\title{
Orientación al mercado en la industria farmacéutica de Venezuela
}

\author{
Márquez, M. Marveya* \\ Molina, Arturo** \\ García, Juan A.**
}

\section{Resumen}

Este estudio presenta un análisis de la orientación al mercado y la influencia del poder y conflicto de los agentes intermediarios de la demanda (prescriptores y dispensadores) sobre los resultados empresariales, realizando una aplicación en la industria farmacéutica de Venezuela. Para su desarrollo se ha realizado una revisión teórica de la orientación al mercado y las fuentes de poder y conflicto en el canal de distribución, y un trabajo de campo entre 223 visitadores médicos de 60 empresas nacionales e internacionales. El contraste de las hipótesis se realizó mediante el análisis de ecuaciones estructurales, en concreto se utilizó la regresión por mínimos cuadrados parciales. El estudio concluye que la orientación al mercado es un concepto multidimensional, que influye positivamente en los resultados empresariales, que no reduce fácilmente las fuentes de poder de los agentes intermediarios de la demanda y que resulta una estrategia adecuada para mitigar el efecto negativo que las fuentes de conflicto con estos agentes tienen sobre los resultados empresariales. Además, si se controlan adecuadamente las relaciones de poder y conflicto en el canal, se obtendrán los resultados empresariales acordes con los objetivos propios y con relación a la competencia. Por tales razones se señala la utilidad que una estrategia de orientación al mercado y la consideración de las fuentes de poder y conflicto en el canal tiene a la hora de mejorar el desempeño de estas empresas.

Palabras clave: Orientación al mercado, fuentes de poder y conflicto, agentes intermediarios, demanda, industria farmacéutica.

\section{Recibido: 25-05-11. Aceptado: 12-04-13}

* Doctora en Creación de Empresas, Dirección de PYMES y Empresa Familiar. Profesora Agregado, Facultad de Farmacia, Universidad de Los Andes, Venezuela, e-mail: marveya@ula.ve.

** Doctor en Ciencias Económicas y Empresariales. Profesor Titular de Universidad, Área de Comercialización e Investigación de Mercados, Facultad de Ciencias Jurídicas y Sociales, Universidad de Castilla-La Mancha, España, e-mail: arturo.molina@uclm.es.

*** Doctorando en Estrategia y Marketing de la Empresa. Profesor Ayudante, Área de Comercialización e Investigación de Mercados, Facultad de Ciencias Jurídicas y Sociales, Universidad de Castilla-La Mancha, España, e-mail: juan.garcia@uclm.es. 


\section{Market Orientation in the Venezuelan Pharmaceutical Industry}

This study presents an analysis of orientation to the market and the influence of power and conflict belonging to the intermediary agents of demand (prescribers and dispensers) on business results, as applied to the Venezuelan pharmaceutical industry. A theoretical review was made about orientation toward the market and the sources of power and conflict in the distribution channel; field work was carried out with 223 medical sales representatives from 60 national and international companies. The contrasting of hypotheses was accomplished through the analysis of structural equations; specifically, partial least squares regression was used. The study concluded that orientation to the market is a multidimensional concept that influences business results positively, that does not easily reduce the sources of power belonging to the intermediary agents of demand, and seems to be an appropriate strategy for mitigating the negative effect that the sources of conflict with these agents have on the business results. Furthermore, if the relations of power and conflict in the channel are adequately controlled, business results will be obtained that agree with the company's objectives and relate to the competition. The study points out the utility of a strategy of orientation to the market and consideration of the sources of power and conflict in the channel for improving the performance of these firms.

Key words: Orientation to the market, sources of power and conflict, intermediary agents, demand, pharmaceutical industry.

\section{Introducción}

Esta investigación se centra en estudiar la orientación al mercado en la industria farmacéutica (IF) y la influencia del poder y conflicto de los agentes intermediarios de la demanda (AID) en la mejora de los resultados empresariales. La selección de esta industria se debe principalmente a su relevancia económica y a su prestigio corporativo a nivel mundial.

De acuerdo con el ranking Fortune Global 500 de 2009, la industria farmacéutica ocupa la tercera posición al considerar el retorno sobre los ingresos (Return on Revenues (ROR)) y sobre los activos (Return on Assets -ROA-), y el quinto lugar en cuanto al retorno a los accionistas (Return on Shareholder -ROS-). Además, atendiendo a sus tasas de crecimiento, ocupa la sexta y vigesimoquinta posición según las variaciones en las ganancias y la facturación, respectivamen- te. Las ventas de la industria farmacéutica mundial, a precios constantes de fabricante, superaron los US\$837 mil millones en 2009 (IMS Health Market Prognosis, 2009). El mayor volumen de ventas se concentra en América del Norte (Estados Unidos y Canadá) y Europa con un $39 \%$ y $31 \%$, respectivamente. En tercer lugar se sitúan Asia, África y Australia (13\%), seguidos de Japón (11\%) y América Latina (6\%). Además, la industria farmacéutica mundial muestra un crecimiento de las ventas del $7 \%$ en el año 2009 , registrándose las tasas de variación más elevadas en Asia, África y Australia ( $16 \%$, de forma conjunta) y América Latina (11\%). Este comportamiento se relaciona con los mercados emergentes de China, Brasil, India, Corea del Sur, México, Turquía y Rusia (denominados Pharmerging) (Burmester, 2010) y con la mejora de la atención sanitaria en determinados países en vías de desarrollo, tales 
como Venezuela (Organización Mundial de la Salud - OMS, 2009a; 2009b).

De forma concreta, este trabajo se centra en la industria farmacéutica de Venezuela. La selección de la industria farmacéutica de este país se debe, entre otros aspectos, a su carácter estratégico en el ámbito nacional y al crecimiento de las ventas experimentado en los últimos años (Organización Mundial de la Salud OMS, 2009a).

Para situar el contexto de la industria farmacéutica y poder comprobar su relevancia en varios aspectos, se pueden señalar sus principales características: complejidad, nivel de competencia, asimetría de información y relaciones con los agentes, regulación y control de precios, Investigación y Desarrollo (I+D), protección de patentes, y gestión del portafolio de medicamentos (Becher y Asociados, 2008). La industria farmacéutica es un importante elemento de los sistemas de asistencia sanitaria en todo el mundo; está constituida por numerosas organizaciones públicas y privadas dedicadas al descubrimiento, desarrollo, fabricación y comercialización de medicamentos para la salud humana y animal (Gennaro, 2008).

Algunas compañías farmacéuticas trabajan tanto en los mercados nacionales como en los multinacionales. La mayoría de los países conceden patentes para los medicamentos o fármacos recientemente desarrollados o modificados, por períodos de unos 15 años a partir de la fecha de autorización. Las compañías asignan una marca registrada a sus innovaciones, que pasan a ser de su propiedad exclusiva. Además, los nuevos medicamentos reciben un nombre genérico oficial de propiedad pública. Una vez que expira la paten- te, cualquier empresa que cumpla las normas del organismo regulador puede fabricar y vender productos con el nombre genérico, lo que supone una amenaza para la empresa que desarrolló el producto patentado. Por otro lado, la industria farmacéutica requiere la inversión de grandes capitales debido a los gastos asociados a la I+D, la autorización de comercialización, la fabricación, la garantía y el control de calidad, la comercialización y las ventas (Spilker, 1994).

En el ámbito académico, en el sector de la industria farmacéutica se han estudiado profusamente aspectos relacionados con I+D (Case, 2010; Kale, 2010), los procesos de producción (Solà i Solà, 2000) y las repercusiones económicas derivadas de la regulación a la que esta industria está sujeta (Nonell y Borrell, 1998). De forma específica, en el campo del marketing, la mayoría de los estudios existentes versan sobre los componentes del Marketing-mix (Frías, 2007). Ahora bien, en la prolífera producción de investigaciones relacionadas con la orientación al mercado, destaca la escasa aplicación de esta teoría en la industria farmacéutica (Reinares y Gutiérrez, 2006; Mahmoud et al., 2010). Trabajos empíricos en el ámbito de la industria farmacéutica justifican el desarrollo de una investigación dirigida a conocer las implicaciones que la teoría de la orientación al mercado puede tener en este tipo de empresas. Adicionalmente, la influencia que ejercen los AID, médicos y farmacéuticos, es muy importante en la industria farmacéutica, ya que éstos son los verdaderos decisores de la demanda del mercado (Frías, 2007). En consecuencia, considerar las relaciones en este canal de distribución ayudaría a comprender el po- 
tencial que la orientación al mercado tiene en la gestión de las diferentes fuentes de poder y conflicto, y en la mejora de los resultados empresariales.

Para desarrollar esta investigación, se plantea un estudio teórico-empírico que aborda la perspectiva de la oferta del mercado del medicamento. En este sentido, se diseñó un modelo de la orientación al mercado en la industria farmacéutica, así como su relación con las fuentes de poder y conflicto y con los resultados empresariales. Tratándose de un estudio desde la perspectiva de la oferta, se decidió utilizar la figura de informante clave (visitadores médicos) para obtener los datos para la contrastación empírica. Para ello, se realizó un muestreo no probabilístico que consideró la población de empresas de la industria farmacéutica en Venezuela, obteniendo finalmente información de 60 empresas distintas con 223 unidades muestrales válidas.

En la conformación definitiva del cuestionario para la recogida de información se incluyeron las aportaciones realizadas por expertos académicos en marketing y los resultados de un pretest realizado a gerentes de la industria farmacéutica. La contrastación de las hipótesis se realizó mediante el análisis de ecuaciones estructurales, en concreto se utilizó la técnica de mínimos cuadrados parciales comúnmente denominada PLS. Entre las principales razones por las que se eligió esta técnica están que el estudio presenta un enfoque relativamente nuevo, así como algunas hipótesis no han sido planteadas en estudios previos y según Reinartz et al. (2009) para una muestra de 223 unidades, esta técnica de PLS muestra mayor potencia estadística.

\section{Relaciones entre orientación al mercado, fuentes de poder y conflicto, y resultados empresariales}

El concepto de orientación al mercado hace referencia a la manera en que la empresa se relaciona, en general, con su mercado y, en particular, con sus consumidores. En el ámbito académico se profundiza en el estudio del concepto de orientación al mercado en los años noventa con los trabajos de Kohli y Jaworski (1990) y Narver y Slater (1990). El primero se sustenta en las actividades de procesamiento de la información del mercado o enfoque del comportamiento organizativo, mientras que el segundo adopta un enfoque cultural, atendiendo principalmente a las normas y valores de la empresa. La inmensa mayoría de los estudios posteriores han versado sobre estos dos enfoques, los cuales han sido ampliados en los últimos años añadiendo a sus definiciones iniciales un matiz proactivo.

Por una parte, el modelo original de Kohli y Jaworski (1990) ha sido ampliado por Jaworski et al. (2000), sugiriendo la existencia de dos aproximaciones complementarias a la orientación al mercado: "estar orientado al mercado" y "orientar los mercados". Ambas están centradas en el consumidor, en los competidores y en las condiciones del mercado. Por otro lado, Narver et al. (2004) señalan que el concepto analizado tiene dos orientaciones complementarias, una reactiva (dirigida a las necesidades actuales de los clientes) y otra proactiva (dirigida a las oportunidades para crear un valor en el consumidor del que actualmente no es consciente). Otro enfoque relevante so- 
bre este aspecto se encuentra en Brickley et al. (2005), quienes señalan la importancia de analizar si la estrategia u orientación de la empresa se ajusta al entorno y al mercado.

La forma de medición de la orientación al mercado debe adaptarse al tipo de empresa y a los objetivos de investigación. Entre los instrumentos para cuantificar el grado de adopción de la orientación al mercado predominan dos: MKTOR (Narver y Slater, 1990) y MARKOR (Kohli et al., 1993). De ellos se derivan innumerables trabajos que integran estas propuestas o que desarrollan nuevos modelos basados en alguno de los dos instrumentos de medida. No obstante, es preciso señalar que la mayoría de las escalas existentes han sido objeto de críticas en cuanto a su base teórica, su contenido o sus propiedades estadísticas. Por tanto, no existe una única escala de medida del constructo orientación al mercado que pueda ser utilizada para cualquier situación, siendo necesaria la adaptación de las escalas disponibles a las circunstancias particulares de cada caso.

La investigación sobre la orientación al mercado en la industria farmacéutica es incipiente, a pesar del potencial que la aplicación de esta teoría tiene en el sector. Las investigaciones previas realizadas por Reinares y Gutiérrez (2006) y Mahmoud et al. (2010) abordan aspectos limitados y, por tanto, se requiere continuar contextualizando este concepto, considerando que el mercado del medicamento presenta características que difieren de cualquier otro. Por ello, dos aspectos para poder aplicar la orientación al mercado en la industria farmacéutica son: 1) profundizar en la caracterización del sector; y 2) ahondar en el conocimiento de los agentes que intervienen en el canal de distribución y sus relaciones con la industria.

La orientación al mercado es un concepto que ha sido aplicado con éxito en diversos sectores y tipos de empresas, así como en distintos contextos. La industria farmacéutica forma parte de los denominados sectores de alta tecnología, caracterizados por la creciente globalización, la aparición de nuevos competidores y la rápida evolución de la tecnología. Por tanto, a priori, cabe esperar que los preceptos de la orientación al mercado tengan aplicación en este tipo de empresas. Sin embargo, existen pocos estudios en los que se analice la aplicación de la orientación al mercado en la industria farmacéutica (Reinares y Gutiérrez, 2006; Mahmoud et al., 2010). A pesar de ello, existen varias razones que llevarían a postular la orientación al mercado como una estrategia adecuada para las empresas que forman parte de esta industria:

- Las empresas de la industria farmacéutica deben mejorar de manera continua sus productos, servicios y procesos para poder competir en mercados cada vez más exigentes y dinámicos (Carvajal, 2005). En este contexto, la aplicación de estrategias de gestión orientadas al mercado repercute en una mayor satisfacción de los pacientes, los AID y los agentes financiadores. En el caso de los pacientes, aplicar un enfoque de orientación al mercado permitirá un mayor desarrollo y adecuación de las alter- 
nativas terapéuticas a las diversas patologías existentes. En relación con los AID, este tipo de orientación de la industria farmacéutica les permitirá contar con mayores alternativas terapéuticas disponibles $\mathrm{y}$, también, potenciará una mayor interacción y colaboración entre la industria farmacéutica y estos profesionales (médicos y farmacéuticos, principalmente). Respecto a los agentes financiadores, si la industria farmacéutica proporciona productos en las dosis y presentaciones adecuadas a los tratamientos, permitirá la reducción de costes en el sistema sanitario y contribuirá a mejorar la salud de la población.

- Narver y Slater (1990) argumentan que el desarrollo de nuevos productos debería derivarse de la interacción entre fabricantes y clientes. Teóricamente, en la medida en que la orientación al mercado facilite estas interacciones, su aplicación en la industria farmacéutica permitirá aumentar las posibilidades de éxito de las innovaciones, tanto radicales (medicamentos nuevos para enfermedades nuevas) como incrementales (medicamentos modificados para obtener mejores resultados, hacerlos más cómodos, entre otras) (Reinares y Gutiérrez, 2006). No obstante, por la gran complejidad de la I+D en la industria farmacéutica y las pocas probabilidades de descubrimiento de nuevas moléculas, parece razonable pensar que la orientación al mercado sería capaz de generar mejores me- dicamentos adaptados a las necesidades de los pacientes (innovaciones incrementales), aunque existen dudas sobre el papel que pudiera tener en el desarrollo de las innovaciones radicales.

- La industria farmacéutica se caracteriza por una elevada competitividad. Algunas de las razones que explican esta situación son el vencimiento de patentes y la incorporación de medicamentos genéricos. Por tanto, a medida que el mercado de la industria farmacéutica tiende a situaciones en las que la oferta es mayor que la demanda, las empresas se verán obligadas a desarrollar estrategias de orientación al mercado para alcanzar un mayor nivel de competitividad.

En el contexto de la industria farmacéutica, Mahmoud et al. (2010) adoptan el enfoque de orientación al mercado como comportamiento organizativo, aunque sin valorar la contribución de cada una de las dimensiones que conforman el concepto. Aplicar el concepto de orientación al mercado a esta industria implica que las empresas necesitan adquirir información de una amplia gama de segmentos (incluyendo médicos, farmacias, hospitales, pacientes, competidores, proveedores, etc.), diseminar esta información en todas las áreas funcionales de la empresa y dar una respuesta adecuada (Reinares y Gutiérrez, 2006). Así, la necesidad de profundizar en el conocimiento de las dimensiones que conforman la orientación al mercado lleva a plantear la siguiente hipótesis: 
H1: La orientación al mercado en la industria farmacéutica es un concepto formado por tres dimensiones: a) generación de información, b) diseminación de información y c) capacidad de respuesta.

Ahora bien, en un sector industrial como la industria farmacéutica, con características tan específicas, la pregunta es: ¿la orientación al mercado será capaz de mejorar sus resultados empresariales? Los argumentos anteriores llevan a pensar que aquellas organizaciones que recopilan, difunden y utilizan más y mejor la inteligencia de mercado serán capaces de obtener mejores resultados. En este sentido, la única investigación empírica encontrada muestra una asociación positiva y significativa entre la orientación al mercado y el rendimiento empresarial (excepto sobre el crecimiento de las ventas) (Mahmoud et al., 2010). Así, considerando el estudio de Avlonitis y Gounaris (1997), que aborda la influencia de la orientación al mercado sobre dos perspectivas de resultados (en relación con los objetivos propios y la competencia), se plantea la siguiente hipótesis:

H2: La orientación al mercado en la industria farmacéutica influye positivamente en los resultados empresariales en relación con: a) los objetivos propios y b) la competencia.

Continuando con otros aspectos vinculados a este estudio (poder y conflicto en los canales de distribución), estos constituyen dos de los tópicos analizados con mayor profundidad en la literatura sobre las relaciones entre el fabricante y el distribuidor (Stern et al., 1999). Respecto al estudio del poder, su importancia radica en que se trata de un factor explicativo en la conformación del canal de distribu- ción, así como de un instrumento de control y coordinación de las funciones comerciales y de las organizaciones que las ejecutan (Múgica, 1985). El poder es clave para explicar las relaciones en el canal (reparto de actividades, rol de cada miembro, entre otras) y, por tanto, determina los resultados alcanzados por los agentes (Cruz et al., 2001).

Para que los productos de la industria farmacéutica y, lo que es aún más importante, sus beneficios para la salud, lleguen a los pacientes es preciso que existan canales de distribución adecuados. Así refiriéndose a la distribución y dispensación de medicamentos, Carroll (2008) señala que los agentes de los canales de distribución participan en la cadena del valor del medicamento mediante dos tipos de actuaciones. Por una parte, están las acciones propias de cualquier sistema de distribución (compra y venta, financiación, logística, almacenamiento, servicio, entre otras) y que son muy similares a las que se llevan a cabo en la comercialización de otros productos, como alimentos, compuestos químicos, calzado, entre otros. Por otro lado, están las funciones genuinamente sanitarias (prescripción y dispensación) que se acogen a las especificidades de una concreta tradición profesional.

La distribución y dispensación del medicamento está configurada por tres grandes actores: las empresas de distribución mayorista (droguerías), la extensa red de oficinas de farmacia, y los servicios farmacéuticos hospitalarios. Además, existen una serie de agentes que interactúan directamente con la demanda (AID) y que son considerados como decisores de la misma (médicos y farmacéuti- 
cos). La participación de los AID es clave en la decisión de compra de los medicamentos, ya que la legislación de la mayoría de los países les concede el poder exclusivo de prescribir medicamentos, en el caso de los médicos, y de dispensarlos, en el de los farmacéuticos.

En este contexto, la estructura de comercialización del medicamento difiere de cualquier otro producto en el mercado, por la existencia del prescriptor que toma la decisión de compra en nombre del consumidor (Tobar, 2008). Esta convivencia entre diferentes grupos, cada uno con sus propios intereses, supone la aparición de nuevos conflictos en el canal del medicamento, adicionales a los de cualquier estructura de comercialización. Así mismo, la existencia de normas sobre la prescripción de medicamentos genéricos aumenta el rol que ocupa el consumidor en la toma de decisiones, pero referido fundamentalmente al precio y no a la búsqueda de productos sustitutos (como sucedería en el caso de productos de consumo masivo). De hecho, ante el desconocimiento del consumidor, puede aumentar la influencia o poder del farmacéutico (como profesional especializado) por su facultad para decidir el medicamento finalmente dispensado al paciente (Carroll, 2008; Wertheimer, 2008).

Ahora bien, la teoría de orientación al mercado plantea que las empresas orientadas al mercado se apropiarán de la información sobre su entorno externo e interno (Jaworski et al., 2002), serán conscientes de la existencia de fuentes de poder y conflicto en el canal y tendrán capacidad para generar las respuestas más apropiadas con el fin de controlar los efectos perjudiciales en el logro de sus objetivos. Por tanto, se puede inferir que las empresas con una gestión orientada al mercado pueden influir sobre las fuentes de poder y conflicto existentes entre los AID y la organización. En consecuencia, se formulan las siguientes hipótesis:

H3: La orientación al mercado en la industria farmacéutica influye en la disminución de las fuentes de poder de los AID en el canal.

H4: La orientación al mercado en la industria farmacéutica influye en la disminución de las fuentes de conflicto con los AID en el canal.

El poder es la capacidad que tiene un integrante de un canal para obligar a otros miembros a hacer lo que en otras condiciones no haría. Es decir, la industria farmacéutica necesitaría contar con fuentes de poder para conseguir la cooperación de los AID. Esta definición de poder está estrechamente relacionada con el grado de dependencia que cada empresa considere en su toma de decisiones (Miquel et al., 2000).

El análisis de las relaciones de poder en el canal de la industria farmacéutica muestra que cada miembro puede contar con distintas fuentes de poder (lo que determinará el nivel máximo de poder ejercido) y, a su vez, también recibe influencias del resto de los miembros del canal. Esta dicotomía entre influencia ejercida y percibida permite determinar la condición relativa de poder de cada miembro, dando lugar a ventajas o desventajas:

- Situación de ventaja relativa. Los fabricantes y AID se encuentran en esta situación, ya que ostentan mayor capacidad para ejercer poder que para 
recibir influencias por parte del resto de agentes. Lógicamente, al ser los dos miembros que cuentan con una mayor cantidad de fuentes de poder se incrementaran las probabilidades de que sus intereses sean contrapuestos, generándose potenciales situaciones de conflicto.

- Situación de desventaja relativa. Los mayoristas y minoristas están en una situación de desventaja respecto al resto de miembros en el canal. Su capacidad para ejercer influencia es limitada, aunque las fuentes de poder que controlan (por ejemplo, el poder de mercado) pueden poseer gran valor en las relaciones del canal.

Referente a la relación entre fuentes de poder de los AID y resultados empresariales, estudios previos indican que aquellas relaciones del canal caracterizadas por asimetrías de poder generan menor confianza y continuidad (Anderson y Weitz, 1989), y tienen efectos negativos sobre la calidad de la relación y los resultados empresariales (Kumar et al., 1995). Por otra parte, los AID son independientes económicamente y autónomos en su toma de decisiones respecto a la industria farmacéutica, mientras que los resultados de la industria farmacéutica dependen de los AID (son los verdaderos decisores de su demanda) (Carroll, 2008; Wertheimer, 2008). En este sentido, los AID pueden hacer uso de su poder de experiencia (condición profesional), de referencia (prescriben y dispensan), de legitimidad (eligen por el paciente), de información (tienen conocimiento de los pacientes y las patologías) y de persuasión (cuentas con ventajas por la asimetría de información) para influir en las decisiones de otros miembros del canal y lograr sus propios objetivos. Esto puede ser contrario al interés de las empresas farmacéuticas y repercutir negativamente sobre sus resultados. Por tanto, se plantea la siguiente hipótesis:

H5: Las fuentes de poder de los AID en el canal influyen negativamente en los resultados empresariales en relación con: a) los objetivos propios y b) la competencia.

La teoría de los canales de distribución indica que siempre hay conflictos en los mismos. Una razón importante es que los miembros del canal tienen objetivos diferentes $y$, en ocasiones contradictorios. El papel que cumple la industria farmacéutica y los AID en el canal de distribución hace que cada uno sea partícipe importante en la ruta a través de la cual los medicamentos recetados se hacen accesibles y disponibles para los clientes finales (pacientes).

Así, en el canal del medicamento existe una brecha significativa entre la industria farmacéutica y las profesiones de médicos y farmacéuticos. La industria farmacéutica, los médicos y los farmacéuticos tienen objetivos contradictorios que se derivan de una diferencia fundamental: la industria farmacéutica es un negocio, mientras que la medicina y la farmacia son profesiones (Carroll, 2008). Como profesiones, a los médicos les ha sido encomendado el derecho exclusivo de prescribir y a los farmacéuticos el de dispensar los medicamentos recetados. En consecuencia, la industria farmacéutica tiene intereses económicos, mientras que, la sociedad espera que los profesionales 
coadyuven a mantener el bienestar de sus pacientes como su prioridad más importante.

Los médicos pueden tener intereses en la equidad de la droga, en los incentivos de las compañías y/o en la propiedad intelectual de sus productos. Ahora bien, estas motivaciones generan conflictos de interés, ya que el compromiso primario del médico con sus pacientes se contrapone al compromiso secundario hacia el medicamento o el incentivo que ofrece la empresa (Stoltzfus, 2010).

Adicionalmente, Banks (2005) señala que estas diferencias de objetivos entre los tres grupos (industria farmacéutica, médicos y farmacéuticos) se acentúan debido al interés de los pacientes por ser tratados con la droga más segura y eficaz, y poder adquirir los medicamentos recetados al mejor precio; mientras que cada fabricante tiene como objetivo promover el uso de su medicamento, la comercialización en cada categoría y maximizar el retorno de la inversión, a través de la venta de sus productos al mejor precio posible.

En el mercado del medicamento regulado, el consumidor no posee los conocimientos suficientes que le permitan tomar decisiones sobre este tipo de productos y, por razones legales, en muchas circunstancias tampoco lo puede hacer. En este contexto, el médico asesora y prescribe, no sólo por ser un profesional con los conocimientos necesarios, sino también por motivos legales. Por esta razón son tan importantes las estrategias comerciales orientadas al profesional tanto de la medicina como de la farmacia. En consecuencia, las empresas de la industria farmacéutica que han elegido compe- tir en el mercado de los genéricos (siendo el precio el eje de su estrategia) deberían orientar también sus acciones al profesional que dispensa (el farmacéutico) y no sólo al que prescribe.

En el caso de los productos de venta libre, el consumidor puede estar influido por la comunicación de los fabricantes, sobre todo en aquellos casos en los que la venta de este tipo de productos se realiza a través de los modelos de autoservicio. No obstante, el consumo sigue generándose mayoritariamente en los puntos de venta especializados, como es el caso de la oficina de farmacia, sugiriendo una preferencia del consumidor por comprar en espacios donde pueda ser asesorado.

Todo esto se traduce en la existencia de un conflicto por controlar la prescripción y dispensación. De ahí, el interés y los esfuerzos realizados por la industria farmacéutica para influir sobre estas dos actividades.

En lo que respecta a la relación entre fuentes de conflicto con los AID y resultados empresariales, en la revisión teórica sobre los canales de distribución relacionada con el poder y conflicto, no existe consenso definitivo. Por un lado, siguiendo la corriente que considera que los conflictos son funcionales, el conflicto mejoraría la comunicación, proporcionaría una mayor congruencia entre los objetivos de los miembros del canal (Morgan y Hunt, 1994) y redundaría positivamente sobre los resultados. No obstante, para que esto ocurra, el conflicto debe ser de nivel reducido o moderado y, además, las partes implicadas deben conocer su existencia y considerarlo en sus decisiones estratégicas como una oportunidad de mejora. 
Por otro lado, la visión disfuncional de los conflictos (Cruz, 1999) supone que los fabricantes piensan en términos del sistema de distribución, mientras que los AID no se ven necesariamente como parte del mismo, sino que se consideran como operadores independientes. En consecuencia, si a los AID les falta una orientación de sistema, serán reacios a comprometer sus propios intereses en defensa de los intereses del canal, lo que afectará negativamente a los resultados empresariales de la industria farmacéutica. En este sentido, se propone la siguiente hipótesis:

H6: Las fuentes de conflicto con los AID en el canal influyen negativamente en los resultados empresariales en relación con: a) los objetivos propios y b) la competencia.

Finalmente, en este trabajo de investigación también se plantea el posible efecto moderador de la orientación al mercado en la relación entre las fuentes de poder y conflicto y los resultados empresariales. En principio, las fuentes de poder y conflicto influyen negativamente en los resultados empresariales. No obstante, asumir que esto siempre es así implica no considerar las decisiones que la empresa puede tomar.

Cuando la empresa está orientada al mercado conocerá las fuentes de poder con que cuentan los AID y podrá compensar su situación de desventaja relativa invirtiendo en fuentes de poder (Santesmases, 1996) (incentivos o apoyos a la investigación de médicos y farmacéuticos). Con ello, la empresa conseguirá la cooperación de estos agentes (Cano, 2006; Stoltzfus, 2010), disminuirá el nivel de poder ejercido por los AID y mitigará su impacto negativo en los resultados de la empresa (Hunt y Nervin, 1974). La idea que subyace en este planteamiento es que colaborar con el agente controlador del canal potenciará los resultados.

Además, la orientación al mercado puede hacer que un conflicto que, a priori es disfuncional, se convierta en funcional. Así, un conflicto latente detectado a tiempo (por ejemplo, mediante una adecuada inteligencia de mercado) puede llegar a ser una oportunidad de mejora de los resultados empresariales, si la empresa diseña e implanta una respuesta al mercado adecuada (Ehie, 2010). Es decir, la orientación al mercado puede impedir que el conflicto estalle y se convierta en virulento.

Por tanto, cuando exista poder y conflicto, desarrollar una estrategia de orientación al mercado hacia el AID puede incrementar su confianza y satisfacción con la empresa y, en consecuencia, minorar el impacto negativo que las fuentes de poder y conflicto ejercen sobre los resultados empresariales. Así, se plantean las siguientes hipótesis:

H7: La orientación al mercado en la industria farmacéutica modera (debilita) la influencia negativa de las fuentes de poder en los resultados empresariales en relación con: a) los objetivos propios y b) la competencia.

H8: La orientación al mercado en la industria farmacéutica modera (debilita) la influencia negativa de las fuentes de conflicto en los resultados empresariales en relación con: a) los objetivos propios y b) la competencia.

El modelo teórico propuesto aparece recogido en el Gráfico 1. En él se muestra que la orientación al mercado está formada por tres dimensiones (generación 


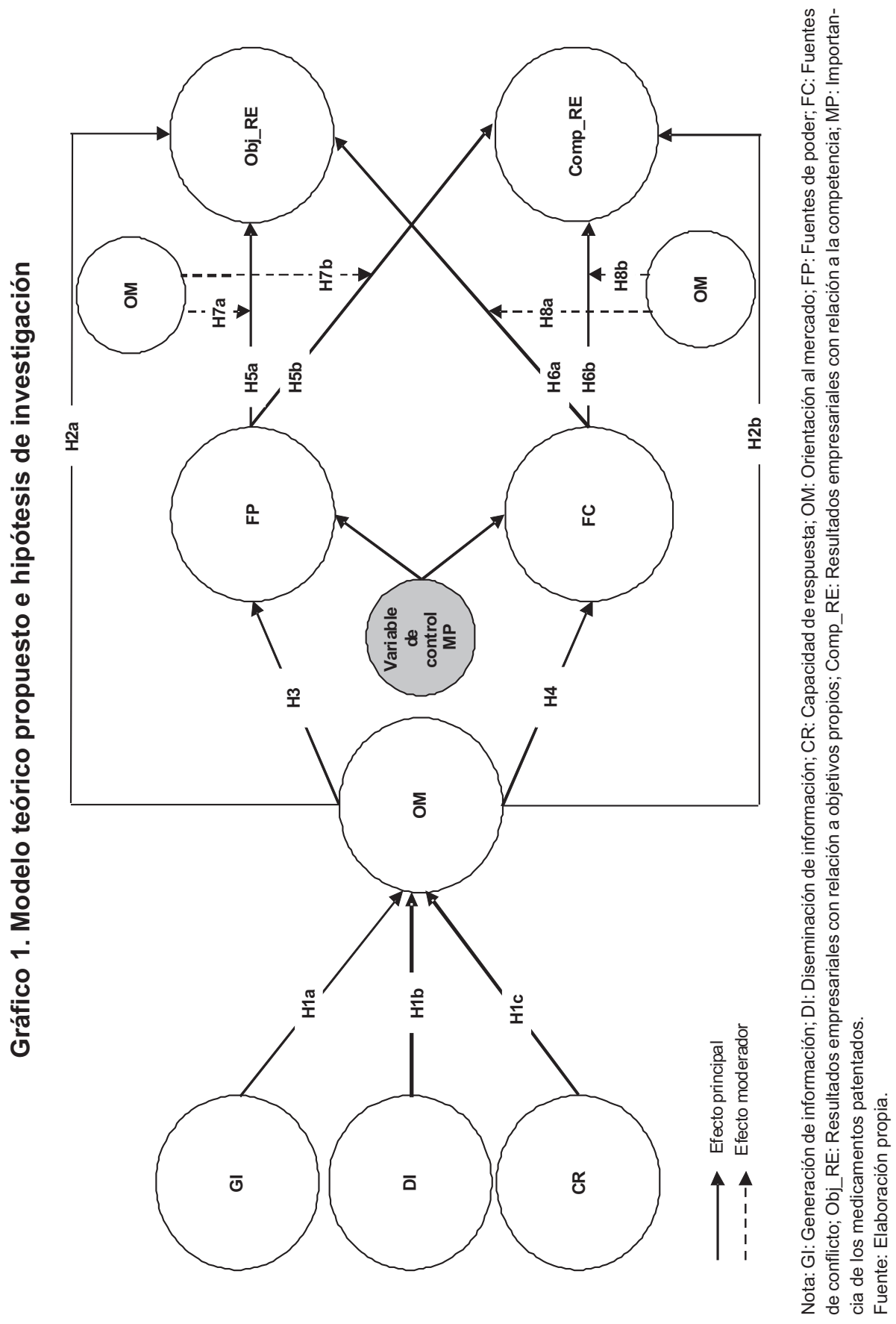


de información, diseminación de información y capacidad de respuesta) $(\mathrm{H} 1 \mathrm{a}-\mathrm{H} 1 \mathrm{c})$. Además, se propone que la orientación al mercado influye sobre los resultados empresariales (con relación a los objetivos propios y a la competencia) a través de tres vías distintas: directamente $(\mathrm{H} 2 \mathrm{a}-\mathrm{H} 2 \mathrm{~b})$, indirectamente (es decir, ejerciendo un efecto mediatizado por las fuentes de poder y conflicto) (H3, H4, H5a-H5b y H6a-H6b), y moderando el impacto negativo de las fuentes de poder y conflicto sobre los resultados (H7a-H7b y H8a-H8b).

Como puede observarse, con el objetivo de controlar que las relaciones planteadas entre la orientación al mercado y las fuentes de poder y conflicto son las verdaderas, se incluyó una variable de control que mide la importancia de los medicamentos patentados en la calidad del servicio, en la posición en el mercado y en las ventas. La introducción de esta variable se debe a que los medicamentos patentados pueden condicionar la existencia de mayores o menores fuentes de poder y conflicto con los AID. De esta forma, su inclusión en el modelo evita que se sobrestime el tamaño de los coeficientes asociados a $\mathrm{H} 3$ y $\mathrm{H} 4$.

\section{Métodos empleados en el estudio empírico}

La muestra objeto de estudio se recogió en Venezuela; participaron 60 empresas de la industria farmacéutica dedicadas a la fabricación de productos medicamentosos en el país. Al tratarse de un estudio de oferta, fue necesario recurrir a la figura del informante clave. En concreto, se decidió que la información de las empresas fuera suministrada por los visitadores médicos por las siguientes razones:

- Son profesionales que trabajan en las compañías farmacéuticas y manejan información general de la empresa.

- Poseen conocimientos científico-técnicos relacionados con los productos que promocionan.

- Conocen las técnicas de ventas aplicadas en el sector farmacéutico.

- Realizan un contacto directo con los prescriptores y dispensadores de medicamentos.

- Son un elemento clave en el proceso de generación de inteligencia, diseminación de información y respuesta al mercado.

Se realizó un muestreo no probabilístico de conveniencia con el objetivo principal de lograr una amplia representación de diferentes empresas. Los datos se recogieron, principalmente, mediante la realización de encuestas personales asistidas $(72 \%)$, aunque también se utilizaron encuestas autoadministradas (en papel a la salida de la visita médica (13\%), online $(11 \%)$ y vía email $(4 \%))$. En total se consiguieron 223 encuestas válidas.

En la medición de los conceptos planteados en el modelo teórico propuesto se utilizaron escalas adaptadas de estudios previos. Así, para medir la orientación al mercado se utilizó una adaptación de la escala MARKOR desarrollada por Kohli et al. (1993) y ampliamente utilizada en investigaciones previas. Se midió a través de 24 ítems seleccionados entre los 32 que componen la escala original. La revisión realizada por los expertos permitió eliminar 8 ítems que no se adaptaban al contexto de la industria farmacéuti- 
ca. Esta escala considera la orientación al mercado como un concepto con tres dimensiones: generación de información sobre el mercado, diseminación de dicha información en la empresa y respuesta al mercado. Otra escala ampliamente utilizada en la literatura es la MKTOR, propuesta por Narver y Slater (1990) y centrada en la perspectiva cultural.

Sin embargo, se consideró que el enfoque comportamental de Kohli et al. (1993) se adaptaba mejor a esta investigación, ya que los informantes clave (visitadores médicos) no manejan información precisa sobre la cultura y decisiones estratégicas, pero sí sobre los aspectos operativos y procedimientos de la empresa.

Por otra parte, se consideró la orientación al mercado como un constructo multidimensional, que estaba integrado por tres dimensiones que recogen un dominio conceptual diferente del constructo agregado (Law et al., 1998). En este sentido, Podsakoff et al. (2006) señalan que un constructo es multidimensional si está constituido por aspectos heterogéneos (cada dimensión realiza una aportación única) y, por tanto, debe ser modelizado como un constructo agregado y no latente.

Siguiendo las recomendaciones de Jarvis et al. (2003) para los constructos de segundo orden, la orientación al mercado se planteó como un modelo tipo II. Es decir, en el primer orden, las dimensiones de generación de información, diseminación de información y capacidad de respuesta fueron medidas como reflejo de sus ítems, mientras que, en el segundo orden, estas tres dimensiones formaban el concepto de orientación al mercado.
Para los constructos con dimensiones formativas no es adecuado emplear la perspectiva de consistencia interna (Bollen, 1989), por lo que se debe examinar cómo se relacionan las medidas con otras variables externas al índice (Diamantopoulos y Winklhofer, 2001). En este sentido, estos autores proponen tres posibilidades: (a) plantear un ítem global que resuma la esencia del contenido que el índice pretende medir; (b) incluir indicadores reflectivos y estimar el ajuste del modelo; y (c) vincular el índice con otros constructos con los que se espera esté relacionado. Atendiendo a estas recomendaciones, en esta investigación se optó por incluir dos ítems globales que resumían el contenido de la orientación al mercado.

Las fuentes de poder se midieron utilizando 7 ítems adaptados de una escala aplicada en la industria agroalimentaria por Ramos y Sanfiel (2006). Para medir las fuentes de conflicto entre los AID y la industria farmacéutica se utilizaron 10 ítems adaptados del estudio realizado en la industria química española por Sanzo y Vázquez (2000).

Los resultados empresariales se han medido considerando aspectos operativos (volumen de ventas y crecimiento de las mismas, y éxito de nuevos productos) y de eficiencia (rentabilidad sobre la inversión, margen de beneficios y rentabilidad global). En esta investigación la medición de los resultados empresariales se efectuó a través de un instrumento subjetivo que permitía evaluar su comportamiento en relación con los objetivos de la empresa ( 6 ítems) y con la competencia (4 ítems). Para todos los indicadores se utilizaron 
escalas de medida formuladas en tipo Likert de cinco posiciones.

Con relación a las técnicas aplicadas en este trabajo de investigación, se utilizó el análisis de ecuaciones estructurales para contrastar las hipótesis, en concreto, la técnica de regresión por mínimos cuadrados parciales (Partial Least Squares - PLS). El software utilizado fue SmartPLS 2.0 (Ringle et al., 2005). Se estimaron dos modelos, uno de efectos principales y otro de efectos moderadores. Existen distintas estrategias que pueden ser utilizadas en PLS para evaluar el efecto moderador. Henseler y Chin (2010) presentan cuatro posibles alternativas o enfoques (producto de los indicadores, en dos etapas, híbrido y de la ortogonalización) que pueden resultar más o menos adecuadas en cada contexto. En esta investigación se pretendía describir el efecto moderador, se contaba con más de 150 unidades muestrales (muchas observaciones) y el número promedio de indicadores por constructo era superior a 6 (muchos indicadores). Por tanto, se utilizó el enfoque del producto de indicadores, ya que en estas circunstancias éste es el más aconsejable (Henseler y Chin, 2010). Además, este enfoque se ha utilizado previamente en otros trabajos que emplean PLS (Chin et al., 1996; 2003; Sánchez et al., 2010).

\section{Orientación al mercado en la industria farmacéutica de Venezuela: evidencias empíricas obtenidas}

En primer lugar, con el objetivo de caracterizar a las empresas, se realizó un análisis descriptivo de los datos obteni- dos. Comenzando por el perfil del encuestado, un $63 \%$ eran visitadores médicos y un $34 \%$ representantes de ventas. Por tanto, el $97 \%$ eran trabajadores de la empresa que interactúan directamente con los AID. El 3\% restante ocupaba otros cargos de responsabilidad en la empresa (director de marketing, jefe de zona, entre otros).

Se obtuvieron datos de 60 empresas distintas. Una cifra bastante elevada si se tiene en cuenta que, de acuerdo con los datos de Ojeda (2009), existen aproximadamente 200 empresas fabricantes de medicamentos en el país. La mayor parte de las empresas participantes estaban ubicadas en Caracas $(70 \%)$ y en el estado de Miranda (16\%), lo que se explica por las características de localización del sector industrial en el país. El $51 \%$ de las empresas superaba los 30 años en el mercado venezolano, el $91 \%$ era de capital privado y el origen del mismo era nacional (en el $45 \%$ de los casos), internacional $(31 \%)$ o mixto $(24 \%)$.

El $52 \%$ de las empresas contaba con más de 1.000 trabajadores, predominando dentro de la plantilla aquéllos con estudios universitarios (70\%). Esto se debe a que la industria farmacéutica en Venezuela está dirigida por individuos con alta formación técnica y requiere de personal altamente especializado en diferentes áreas de conocimiento (Poggi, 2002).

Atendiendo al volumen de ventas en unidades tributarias (UT), el $55 \%$ eran empresas de tamaño medio (entre 100.001 y 250.000 UT). En cuanto a su desempeño, en 2008 la mayor parte de las empresas reportaron, un margen de beneficios, un crecimiento de las ventas 
totales y un crecimiento de la cuota de mercado de hasta el $20 \%$.

La mayoría de las empresas tenía una cobertura nacional del mercado (74\%). Atendiendo al tipo de mercado, en el portafolio de las empresas encuestadas predominaban los medicamentos sin prescripción (55\%), mientras que, según el tipo de medicamento, el mayor porcentaje se registraba en el caso de los genéricos $(70 \%)$. Esta última circunstancia se explica porque en Venezuela los principales medicamentos patentados son importados (Burmester, 2010). No obstante, la mayor parte de las empresas reconocen la importancia que los medicamentos patentados tienen para incrementar sus ventas (58\%), mejorar la calidad del servicio prestado (54\%) y aumentar la participación en el mercado (55\%).

Antes de proceder a contrastar las hipótesis planteadas, se realizó la evaluación del instrumento de medida, verificando su fiabilidad, y validez convergente y discriminante.

En primer lugar, para la evaluación de la fiabilidad se utilizaron dos indicadores: Alpha de Cronbach $(\alpha)$ (Cronbach, 1951) y fiabilidad compuesta (CR) (Werts et al., 1974). Los valores de ambos índices superaban el valor mínimo requerido de 0,7 . Por otra parte, para garantizar la validez convergente se eliminaron aquellos ítems que no eran significativamente distintas de cero y superiores a 0,7. También la varianza extraída promedio (AVE) (Fornell y Larcker, 1981), superaba el valor de 0,5 (Tabla 1).

Para garantizar la validez discriminante, Fornell y Larcker (1981) sugieren que la varianza extraída promedio de cada variable latente debe ser superior al cuadrado de la correlación con cualquier otro constructo. Como se puede observar en la Tabla 2, los elementos mostrados en la diagonal eran mayores que los valores para las correspondientes filas y columnas. Además, se comprobó que las cargas de cada ítem eran superiores a las cargas cruzadas con el resto de variables latentes del modelo (Götz et al., 2010). Por tanto, no existían problemas de validez discriminante.

Por tanto, a la vista de los adecuados resultados estadísticos obtenidos en relación con el modelo medida (Tablas 1 y 2), este estudio proporciona a los gerentes de la industria farmacéutica una serie de instrumentos fiables y válidos que pueden ser utilizados en estudios internos para realizar sus propias mediciones: del grado en que su empresa está orientada al mercado, así como sobre la existencia de fuentes de poder y conflicto en los distintos canales utilizados por la empresa para la distribución de sus productos.

Dado que en esta investigación se trataba de analizar la orientación al mercado y las diferentes influencias que el poder y conflicto (que ostentan los AID) ejerce en la mejora de los resultados empresariales, fue necesario considerar tanto los efectos principales de dichas relaciones como los efectos moderadores de la orientación al mercado, estimándose, por tanto, dos modelos que son analizados a continuación: uno con los efectos principales y otro que incorporaba los efectos moderadores.

En PLS el modelo estructural es evaluado basándose en dos criterios: el coeficiente de determinación $\left(R^{2}\right)$ y el test de Stone-Geisser $\left(Q^{2}\right)$ (Stone, 1974; Geisser, 1975). En ambos modelos, los 
Orientación al mercado en la industria farmacéutica de Venezuela

Márquez, M. Marveya; Molina, Arturo y García, Juan A.

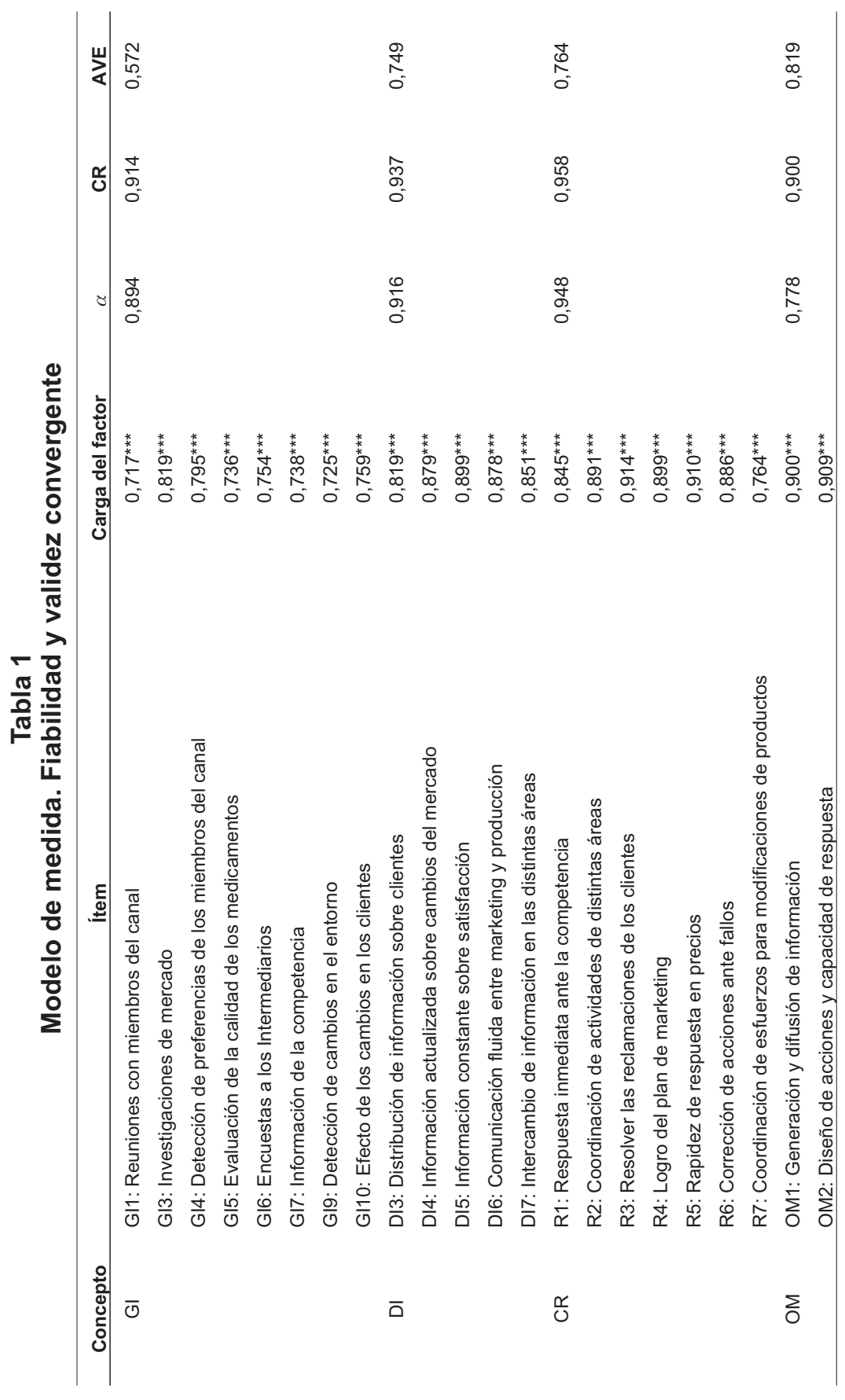




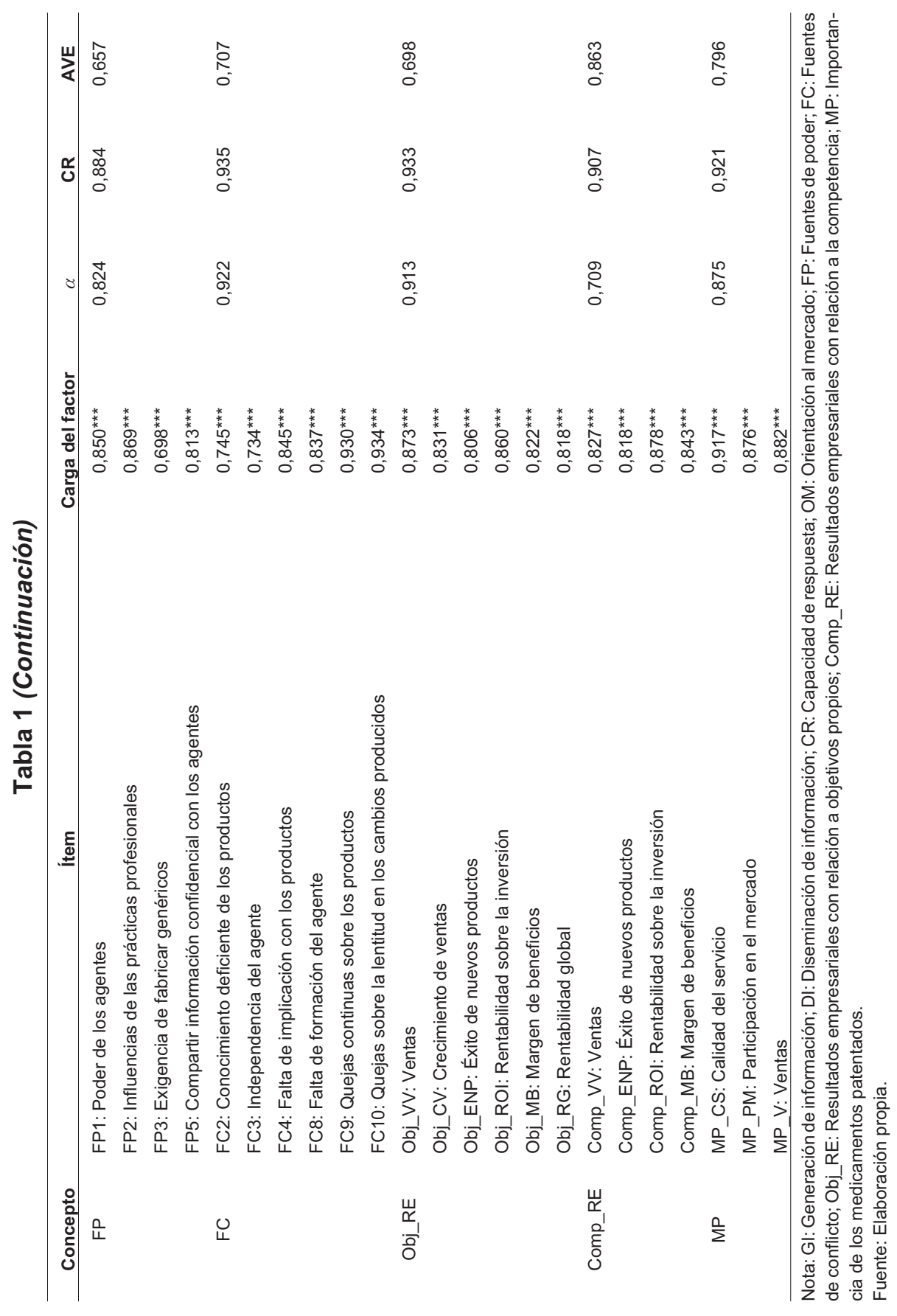


Orientación al mercado en la industria farmacéutica de Venezuela

Márquez, M. Marveya; Molina, Arturo y García, Juan A.

Tabla 2

Modelo de medida. Validez discriminante

\begin{tabular}{cccccccccc}
\hline Concepto & GI & DI & CR & OM & FP & FC & Obj_RE & Comp_RE & MP \\
\hline GI & 0,572 & & & & & & & & \\
DI & 0,396 & 0,749 & & & & & & & \\
CR & 0,298 & 0,582 & 0,764 & & & & & & \\
OM & 0,427 & 0,492 & 0,460 & 0,819 & & & & & \\
FP & 0,185 & 0,052 & 0,074 & 0,073 & 0,657 & & & & \\
FC & 0,082 & 0,017 & 0,077 & 0,020 & 0,523 & 0,707 & & & \\
Obj_RE & 0,344 & 0,548 & 0,536 & 0,352 & 0,050 & 0,038 & 0,698 & & \\
Comp_RE & 0,281 & 0,427 & 0,469 & 0,303 & 0,046 & 0,057 & 0,590 & 0,709 & \\
MP & 0,358 & 0,310 & 0,305 & 0,283 & 0,110 & 0,092 & 0,525 & 0,579 & 0,796 \\
\hline
\end{tabular}

Nota: Diagonal: varianza extraída promedio; Debajo de la diagonal: correlación al cuadrado entre los conceptos; GI: Generación de información; DI: Diseminación de información; CR: Capacidad de respuesta; OM: Orientación al mercado; FP: Fuentes de poder; FC: Fuentes de conflicto; Obj_RE: Resultados empresariales con relación a objetivos propios; Comp_RE: Resultados empresariales con relación a la competencia; MP: Importancia de los medicamentos patentados.

Fuente: Elaboración propia.

indicadores $R^{2}$ y $Q^{2}$ mostraron niveles adecuados, excepto el $R^{2}$ asociado a las fuentes de conflicto cuyo valor $(9,3 \%)$ es ligeramente inferior al mínimo requerido del $10 \%$. Además de los dos criterios anteriores, Tenenhaus et al. (2005) desarrollan un índice para evaluar la bondad de ajuste del modelo (GoF) que se calcula como la media geométrica del promedio del AVE y del promedio del $R^{2}$. Como se puede comprobar, en estos dos modelos este índice superaba el valor recomendado de 0,36 , sugiriendo una bondad de ajuste elevada (Sánchez et al., 2010) (Tabla 3$)$.

Una vez garantizada la calidad de los modelos, se procedió a contrastar las hipótesis, las seis primeras a partir del modelo con los efectos principales y las dos últimas empleando el modelo con los efectos moderadores (Tabla 4). Comenzando por $\mathrm{H} 1$, los resultados mostraron que la orientación al mercado se formaba de manera positiva y significativa por las dimensiones de generación de información $(\beta=0,319 ; p<0,01)$, diseminación de información $(\beta=0,278 ; p<0,01)$ y capacidad de respuesta $(\beta=0,292 ; p<0,01)$, no rechazándose H1. Por lo tanto, la orientación al mercado en la industria farmacéutica es un concepto formado por tres dimensiones: generación de información, diseminación de información y capacidad de respuesta.

Si la gerencia de cualquier empresa que opere en este sector desea mejorar su grado de orientación al mercado tendrá que trabajar en el diseño e implantación de estos tres aspectos. En primer lugar, focalizar la atención en la generación de información sobre los miembros del canal e intermediarios, las estrategias y acciones puestas en marcha por la competencia, las preferencias de los 
Tabla 3

Evaluación de los modelos estimados

\begin{tabular}{ccccccc}
\hline \multirow{2}{*}{ Concepto } & \multicolumn{2}{c}{$\begin{array}{c}\text { Modelo con los } \\
\text { efectos principales }\end{array}$} & & \multicolumn{2}{c}{$\begin{array}{c}\text { Modelo con los } \\
\text { efectos moderadores }\end{array}$} \\
\cline { 2 - 3 } \cline { 6 - 7 } & $\mathbf{R}^{2}$ & $\mathbf{Q}^{2}$ & & $\mathbf{R}^{2}$ & $\mathbf{Q}^{2}$ \\
\hline OM & $60,1 \%$ & 0,482 & & $60,1 \%$ & 0,482 \\
FP & $12,2 \%$ & 0,070 & & $12,2 \%$ & 0,070 \\
FC & $9,3 \%$ & 0,054 & & $9,3 \%$ & 0,054 \\
Obj_RE & $36,5 \%$ & 0,247 & & $37,1 \%$ & 0,248 \\
Comp_RE & $33,4 \%$ & 0,228 & & $37,1 \%$ & 0,251 \\
\hline Índice de bondad del ajuste (GoF) & \multicolumn{3}{c}{0,467} & & \multicolumn{3}{c}{0,442} \\
\hline
\end{tabular}

Nota: OM: Orientación al mercado; FP: Fuentes de poder; FC: Fuentes de conflicto; Obj_RE: Resultados empresariales con relación a objetivos propios; Comp_RE: Resultados empresariales con relación a la competencia.

Fuente: Elaboración propia.

Tabla 4

Hipótesis y coeficientes path

\begin{tabular}{|c|c|c|c|}
\hline Hipótesis & $\begin{array}{l}\text { Modelo con los } \\
\text { efectos principales }\end{array}$ & $\begin{array}{l}\text { Modelo con los } \\
\text { efectos moderadores }\end{array}$ & Conclusión \\
\hline H1a: GI->OM & $0,319^{* * *}$ & $0,319^{\star * *}$ & No rechazar \\
\hline H1b: DI->OM & $0,278^{* * *}$ & $0,278^{\star * *}$ & No rechazar \\
\hline H1c: CR->OM & $0,292^{* * *}$ & $0,292^{* * *}$ & No rechazar \\
\hline H2a: OM->Obj_RE & $0,583^{* * *}$ & $0,569^{* * *}$ & No rechazar \\
\hline H2b: OM->Comp_RE & $0,546^{* * *}$ & $0,560^{* * *}$ & No rechazar \\
\hline H3: OM->FP & $-0,130^{*}$ & $-0,130^{* *}$ & No rechazar \\
\hline $\mathrm{H} 4: \mathrm{OM}->\mathrm{FC}$ & $0,030^{\text {n.s }}$ & $0,030^{n . s}$ & Rechazar \\
\hline H5a: FP->Obj_RE & $0,035^{\mathrm{n} . \mathrm{s}}$ & $0,038^{n . s}$ & Rechazar \\
\hline H5b: FP->Comp_RE & $0,106^{n . s}$ & $0,081^{n . s}$ & Rechazar \\
\hline H6a: FC->Obj_RE & $-0,139^{*}$ & $-0,119^{*}$ & No rechazar \\
\hline H6b: FC->Comp_RE & $-0,239^{\star * *}$ & $-0,195^{\star \star}$ & No rechazar \\
\hline H7a: FP*OM->Obj_RE & & $-0,078^{n . s}$ & Rechazar \\
\hline H7b: FP*OM->Comp_RE & & $-0,231^{* * *}$ & Rechazar \\
\hline H8a: FC*OM->Obj_RE & & $-0,010^{\text {n.s }}$ & Rechazar \\
\hline H8b: FC*OM->Comp_RE & & $0,164^{*}$ & No rechazar \\
\hline Relación de control: MP->FP & $-0,263^{* \star *}$ & $-0,263^{\star * *}$ & - \\
\hline Relación de control: MP->FC & $-0,319^{* * *}$ & $-0,319^{* * *}$ & - \\
\hline \multicolumn{4}{|c|}{$\begin{array}{l}\text { Nota: }{ }^{* *} \mathrm{p}<0,01 ;{ }^{* *} \mathrm{p}<0,05 ;{ }^{*} \mathrm{p}<0,1 ;{ }^{\mathrm{n} . \mathrm{s}} \text { no significativo; GI: Generación de información; DI: Diseminación de informa } \\
\text { ción; CR: Capacidad de respuesta; OM: Orientación al mercado; FP: Fuentes de poder; FC: Fuentes de conflict } \\
\text { Obj_RE: Resultados empresariales con relación a objetivos propios; Comp_RE: Resultados empresariales co } \\
\text { relación a la competencia; MP: Importancia de los medicamentos patentados. } \\
\text { Fuente: Elaboración propia. }\end{array}$} \\
\hline
\end{tabular}


clientes, y los cambios en el entorno. La realización de investigaciones de mercado y reuniones con miembros del canal será muy relevante a la hora de genera dicha información. En segundo lugar, será necesario que esa información se disemine internamente dentro de la empresa (poniendo en marcha un adecuado sistema de información de marketing) para que esté a disposición de los responsables de la toma de decisiones en los distintos ámbitos. En tercer lugar, la empresa tendrá que trabajar para observar y dar respuesta de forma sistemática a las reclamaciones de los clientes, corregir los fallos detectados, entre otros.

Respecto a las consecuencias de la orientación al mercado sobre los resultados, en relación con los objetivos propios $(\beta=0,583 ; p<0,01)$ y la competencia $(\beta=0,546 ; p<0,01)$, se comprobó que existía una relación positiva, proporcionando soporte empírico para no rechazar H2. En otras palabras, la orientación al mercado en la industria farmacéutica influye positivamente en los resultados empresariales en relación con los objetivos propios y de la competencia. Esta circunstancia conduce a una implicación práctica para la gerencia, revelando que aquellas empresas que consiguen desarrollar una adecuada orientación al mercado tienen mayores posibilidades de tener éxito y mejorar sus resultados empresariales.

En cuanto a la relación entre la orientación al mercado y las fuentes de poder y conflicto, se comprobó que el grado de orientación al mercado se relacionaba de forma inversa y significativa con las fuentes de poder $(\beta=-0,130 ; p<0,1)$, mientras que la orientación al mercado no ejercía influencia significativa sobre las fuentes de conflicto $\beta=0,030 ;$ n.s), por tanto, no se rechazó H3, pero sí H4. En este caso, la orientación al mercado en la industria farmacéutica influye en la disminución de las fuentes de poder de los AID en el canal, pero no de los conflictos. En este sentido, sabiendo que las empresas pueden reducir las fuentes de poder que poseen los AID mediante la orientación al mercado, la gerencia podrá centrar sus esfuerzos en identificar la existencia de grupos poderosos AID, entablar relaciones más sólidas con estos grupos, e identificar nuevos grupos de AID.

Las fuentes de poder no tenían un impacto significativamente distinto de cero sobre los resultados en relación con los objetivos propios $(\beta=0,035 ;$ n.s $)$ y la competencia $(\beta=0,106 ; \mathrm{n} . \mathrm{s})$, rechazándose H5. En consecuencia, las fuentes de poder de los AID en el canal no influyen negativamente en los resultados empresariales en relación con los objetivos propios y de la competencia. Sin embargo, las fuentes de conflicto sí ejercen una influencia negativa sobre los resultados en relación con los objetivos propios y de la competencia $(\beta=-0,139 ; \mathrm{p}<0,1 ; \mathrm{y} \quad \beta=$ $-0,239 ; p<0,01$, respectivamente), no rechazándose H6. En relación con el efecto de la variable control, importancia de los medicamentos patentados, ésta se relacionaba de manera inversa con las fuentes de poder $(\beta=-0,262 ; p<0,01)$ y conflicto $(\beta=-0,319 ; p<0,01)$, tal y como se esperaba a priori.

Por otro lado, se analizó el modelo con los efectos moderadores para contrastar $\mathrm{H} 7$ y H8. Comenzando por $\mathrm{H} 7 \mathrm{a}$, el coeficiente asociado no era distinto de cero $(\beta=-0,078 ; n$.s) y, en consecuencia, 
no existía el efecto moderador de la orientación al mercado entre las fuentes de poder y los resultados en relación con los objetivos propios. En cuanto a $\mathrm{H} 7 \mathrm{~b}$, aunque el efecto moderador sí es distinto de cero $(\beta=-0,231 ; p<0,01)$, éste es de signo contrario al esperado, ya que un incremento de la orientación al mercado (en una unidad respecto a la media) provoca que el impacto de las fuentes de poder en los resultados empresariales en relación con la competencia sea negativo (pasando de 0,081 a $-0,150)$. Por tanto, se rechazó $\mathrm{H} 7$ en su conjunto.

En lo referente al efecto moderador que la orientación al mercado tiene en la relación entre fuentes de conflicto y resultados empresariales con respecto a los objetivos propios, se rechazó H8a, puesto que el parámetro asociado a este efecto interacción no era significativamente distinto de cero $(\beta=-0,010 ;$ n.s). Respecto a H8b, no se rechazó esta hipótesis al obtenerse un parámetro cuasi-significativo $(\beta=0,164 ; p<0,1)$. Es decir, la orientación al mercado debilitaba la influencia negativa que las fuentes de conflicto ejercían sobre los resultados empresariales según la perspectiva de la competencia (pasando de $-0,195$ a -0,031).

Ante estos resultados, es necesario que los gerentes sean conscientes del impacto tan negativo que los conflictos con los AID tienen sobre los resultados empresariales, con el fin de propiciar estrategias que mejoren las relaciones entre estos agentes y la empresa. De esta forma, una adecuada orientación al mercado en la industria farmacéutica, centrándose en la generación y diseminación de información, así como en la capacidad de respuesta, resultará beneficiosa para atenuar el impacto negativo que las fuentes de conflicto tienen sobre los resultados, mejorando el desempeño de la empresa respecto al de sus competidores.

Finalmente, se calculó el tamaño del efecto en aquellos casos en lo que el parámetro asociado al efecto interacción era significativamente distinto de cero. Utilizando el $\mathrm{f}^{2}$ propuesto por Cohen (1988), el efecto moderador de la orientación al mercado en las relaciones entre fuentes de poder y conflicto y resultados empresariales desde la perspectiva de la competencia era débil $\left(f^{2}=0,059\right)$. No obstante, es preciso considerar que un $\mathrm{f}^{2}$ reducido no implica necesariamente que no deba ser analizado (Chin et al., 2003).

\section{Conclusiones}

Esta investigación contribuye a ampliar el conocimiento académico que existe actualmente sobre un tema poco investigado en la industria farmacéutica: la orientación al mercado. Desde una vertiente teórica, este artículo contribuye a completar la literatura académica existente sobre la conceptualización de la orientación al mercado, en este caso como un constructo multidimensional formado por tres elementos (generación de información, diseminación de información y capacidad de respuesta). Adicionalmente, teniendo en cuenta que la mayoría de las investigaciones sobre orientación al mercado no han considerado los efectos que pueden ejercer los intermediarios sobre los resultados empresariales, la aplicación empírica realizada en este trabajo con los AID ha permitido contrastar hipótesis no testadas previamente. Por tanto, este estudio aporta un análi- 
sis que permite establecer aportaciones teóricas e interesantes recomendaciones estratégicas.

Con carácter general, se comprueba que la aplicación de estrategias de gestión orientadas al mercado permite conseguir mayores resultados empresariales desde la perspectiva de los objetivos propios y de la competencia. No obstante, a partir de la aplicación realizada en este estudio, se concluye que la orientación al mercado resulta ser una estrategia débil si se pretenden reducir las fuentes de poder que existen con los AID en el canal de distribución, sobre todo cuando los medicamentos patentados tienen gran importancia. Es decir, ante situaciones de preponderancia de este tipo de medicamentos, los esfuerzos en inteligencia de mercado pueden ser nulos o incluso contraproducentes. Adicionalmente, la orientación al mercado es capaz de minorar el efecto negativo que las fuentes de conflicto ejercen en los resultados empresariales en relación con la competencia.

A pesar de que el mercado de la industria farmacéutica está constituido en su mayoría por productos de necesidad vital y, por tanto, las empresas requerirían sólo algunas actividades de orientación al mercado, es cierto que la complejidad competitiva del sector supone que cuanto mayor sea el nivel de orientación al mercado mayor será el rendimiento de estas empresas, ya que podrán tener controladas las potenciales fuentes de conflicto.

Por otro lado, se puede señalar que las fuentes de poder de los AID en el canal de distribución no influyen sobre los resultados empresariales de la industria farmacéutica, mientras que las fuentes de conflicto sí tienen un efecto negativo en los resultados de las empresas de este sector. Este efecto incide en lo perjudicial que puede resultar el desarrollo de continuos conflictos entre la empresa y los AID, ya que la propia empresa disminuye sus resultados $y$, al no lograr la satisfacción de los AID, sus preferencias se pueden desplazar hacia los competidores. Sin embargo, esto no implica que siempre se deba evitar el conflicto, sino más bien hay que buscar la manera de optimizar sus efectos sobre los resultados empresariales.

En esta investigación se ha analizado la influencia de la orientación al mercado en la industria farmacéutica; manifestando que aquellas empresas que consigan desarrollar una adecuada orientación al mercado lograrán tener mejores resultados empresariales. Por otra parte, de acuerdo a este estudio, la orientación al mercado y las relaciones de poder y conflicto que se desarrollan en el canal son el eje básico para la consecución de resultados empresariales acordes con los objetivos propios y en relación con la competencia. Por estas razones, se evidencia que la industria farmacéutica debe orientar sus estrategias hacia dos metas fundamentales: a) potenciar la orientación al mercado en el seno de las organizaciones empresariales y b) vigilar las relaciones de poder y conflicto dentro de los canales de distribución.

Adicionalmente, una adecuada orientación al mercado, centrándose en la efectividad y eficiencia terapéutica del producto y en el mayor desarrollo de I+D, puede resultar beneficiosa para reducir los conflictos con los AID. Asimismo, es necesario que las empresas detecten la existencia de grupos emergentes e insa- 
tisfechos de AID, con el fin de establecer nuevas relaciones con estos dos grupos, ampliar su ámbito de acción y beneficiarse de los nuevos centros de poder.

Por consiguiente, en la medida en que la orientación al mercado facilite la interacción entre empresas y agentes del mercado, su aplicación en la industria farmacéutica permitirá aumentar las posibilidades de éxito de las innovaciones.

\section{Referencias bibliográficas}

Anderson, Erin y Weitz, Barton (1989). Determinants of continuity in conventional industrial channel dyads, Marketing Science, Vol. 8, No. 4, United States, Informs, pp 310-323.

Avlonitis, George J. y Gounaris, Spiros P. (1997). Marketing orientation and company performance: industrial vs. consumer goods companies, Industrial Marketing Management, $\mathrm{Vol} 26$, No 5, Netherlands, Elsevier, pp 385402.

Banks, David (2005). Pharmacists, pharmaceutical manufacturers, and conflicts of interest, American Journal of Health-System Pharmacy, Vol 62, No 17, United States, American Society of Health-System Pharmacists, pp 1827-1832.

Becher y Asociados S.R.L. (2008). Laboratorios e industria farmacéutica, Reporte Sectorial, Año 1, No 2, Argentina, Becher y Asociados S.R.L., pp 1-63.

Bollen, Kenneth A. (1989). Structural Equations with Latent Variables, WileyBlackwell, New York.

Brickley, James A. Smith, Clifford W. y Zimmerman, Jerold L. (2005). Economía Empresarial y Arquitectura de la Organización, McGraw-Hill, Madrid.

Burmester, José (2010). Mercados farmacéuticos emergentes representan el $48 \%$ de crecimiento del mercado en 2013, Blog Bitácora Farmacéutica, en línea: http://bitacorafarmaceutica. wordpress.com/2010/

04/14/mercados-farmaceuticos-emer gentes-representan-el-48-de-crecimi ento-del-mercado-en-2013, fecha de consulta: 09.09.2010.

Cano, Alfredo (2006). Uso racional de fármacos. Influencias y conflictos en la prescripción, en Asociación Española de Pediatría y Atención Primaria (ed.), Actualización en Pediatría 2006, Madrid, pp 77-89.

Carroll, Norman V. (2008). Examining the rift between the pharmaceutical industry and the pharmacy profession: a channels of distribution approach, Journal of Pharmaceutical Marketing \& Management, Vol. 18, No. 2, United States, Haworth Press, pp 21-33.

Carvajal, Oswaldo Raphael (2005). Futuro de las fusiones en la industria farmacéutica mundial, Negotium, Vol 1, No 1, Venezuela, Fundación Miguel Unamuno y Jugo, pp 12-34.

Case, Randolph H. (2010). Managing risk in pharmaceutical R\&D, Research Technology Management, Vol 53, No 2, United Sates, Industrial Research Institute, pp 24-32.

Chin, Wynne W.; Marcolin, Barbara L. y Newsted, Peter R. (1996). A partial least squares latent variable modeling approach for measuring interaction effects: results from a monte carlo simulation study and voice mail emotion/adoption study, en J.I. DeGross, S. Jarvenpaa y A. Srinivasan (eds.), Seventeenth International Conference on Information Systems, Cleveland, pp 21-41.

Chin, Wynne W., Marcolin, Barbara L. y Newsted, Peter R. (2003). A partial least squares latent variable modeling approach for measuring interaction effects: results from a monte carlo si- 
mulation study and voice mail emotion/adoption study, Information Systems Research, Vol 14, No 2, United States, Institute for Operations Research and the Management Sciences, pp 189-217.

Cohen, Jacob (1988). Statistical Power Analysis for the Behavioral Sciences, Lawrence Erlbaum, Hillsdale.

Cronbach, Lee J. (1951). Coefficient alpha and the internal structure of tests, Psychometrika, Vol. 16, No. 3, Canada, Psychometric Society, pp 297-334.

Cruz, Ignacio (1999). Los Canales de Distribución de Productos de Gran Consumo: Concentración y Competencia, Pirámide, Madrid.

Cruz, Ignacio; Yagüe, M. Jesús; Rubio, Luis y Oubiña, Javier (2001). La valoración de los niveles de poder ejercido y conflicto percibido en los canales de distribución de productos de gran consumo, Estudios sobre Consumo, No 57, España, Instituto Nacional de Consumo, pp 10-31.

Diamantopoulos, Adamantios y Winklhofer, Heidi M. (2001). Index construction with formative indicators: an alternative to scale development, Journal of Marketing Research, Vol 38, No 2, United States, American Marketing Association, pp 269-277.

Ehie, Ike C. (2010). The impact of conflict on manufacturing decisions and company performance, International Journal of Production Economics, Vol 126, No 2, Netherlands, Elsevier, pp 145-157.

Fornell, Claes y Larcker, David F. (1981). Evaluating structural equations models with unobservable variables and measurement error, Journal of Marketing Research, Vol 18, No 1, United States, American Marketing Association, pp 39-50.

Fortune Global 500 (2009). Global 500, our annual ranking of the world's largest cor- porations, Revista Fortune, United States, Time Inc, en línea: http://money.cnn.com/magazines/fortune/fortune500, fecha de consulta: 10.02. 2010.

Frías, Dolores M. (2007). Marketing Farmacéutico, Pirámide, Madrid.

Geisser, Seymour (1975). The predictive sample reuse method with applications, Journal of the American Statistical Association, Vol 70, No 350, Unites States, American Statistical Association, pp 320-328.

Gennaro, Alfonso (2008). Remington's Pharmaceutical Sciences, Mack Publishing Company, Easton.

González, Eduardo (2008). Política farmacéutica saludable, Salud Pública de México, Vol. 50, No 4, Cuernavaca, pp. 488-495.

Götz, Oliver; Liehr-Gobbers, Kerstin y Krafft, Manfred (2010). Evaluation of structural equation models using the partial least squares (PLS) approach en V. Esposito Vinzi, W.W. Chin, J. Henseler y $\mathrm{H}$. Wang (eds.), Handbook of Partial Least Squares: Concepts, Methods and Applications, Springer, Heidelberg, pp 691-711.

Henseler, Jörg y Chin, Wynne W. (2010). A comparison of approaches for the analysis of interaction effects between latent variables using partial least squares path modeling, Structural Equation Modeling, Vol 17, No 1, Unites States, Lawrence Erlbaum, pp 82-109.

Hunt, Shelby D. y Nevin, John (1974). Power in a channel of distribution: sources and consequences, Journal of Marketing Research, Vol 11, No 2, United States, American Marketing Association, pp 186-193.

IMS Health Market Prognosis (2009). Total Unaudited and Audited Global Pharmaceutical Market, 2002-2009, en línea: http://www.imshealth.com, fecha de consulta: 12.04.2010. 
Jarvis, Cherly Burke; MacKenzie, Scott B. y Podsakoff, Philip M. (2003). A critical review of construct indicators and measurement model misspecification in marketing and consumer research, Journal of Consumer Research, Vol 30, No 2, United States, University of Chicago Press, pp 199-218.

Jaworski, Bernard J., Kohli, Ajay K. y Sahay, Arvind (2000). Market-driven versus driving markets, Journal of the Academy of Marketing Science, Vol 28, No 1, United States, Sage, pp 45-54.

Jaworski, Bernard J., Macinnis, D.J. y Kohli, Ajay K. (2002). Generating competitive intelligence in organizations, Journal of Market-Focused Management, Vol. 5, No. 4, Unites States, Kluwer Academics, pp 279-307.

Kale, Dinar (2010). The distinctive patterns of dynamic learning and inter-firm differences in the Indian pharmaceutical industry, British Journal of Management, Vol. 21, No. 1, United Kingdom, Blackwell, pp 223-238.

Kohli, Ajay K. y Jaworski, Bernard J. (1990). Market orientation: the construct, research propositions, and managerial implications, Journal of Marketing, Vol. 54, No. 2, United States, American Marketing Association, pp 1-18.

Kohli, Ajay K.; Jaworski, Bernard J. y Kumar, Ajith (1993). MARKOR: a measure of market orientation, Journal of Marketing Research, Vol 30, No 4, United States, American Marketing Association, pp 467-477.

Kumar, Nirmalya; Scheer, Lisa K. y Steenkamp, Jan-Benedict E.M. (1995). The effects of perceived interdependence on dealer attitudes, Journal of Marketing Research, Vol. 32, No. 3, United States, American Marketing Association, pp 348-356.

Law, Kenneth S.; Wong, Chi-Sun y Mobley, William H. (1998). Toward taxonomy of multidimensional constructs, Academy of Management Review, Vol. 23, No. 4, Unites States, Academy of Management, pp 741-745.

Mahmoud, Mohammed A.; Kastner, Adelaide y Yeboah, Joseph (2010). Antecedents, environmental moderators and consequences of market orientation: $A$ study of pharmaceutical firms in Ghana, Journal of Medical Marketing, Vol. 10, No. 3, United Kingdom, Palgrave Macmillan, pp 231-244.

Miquel, Salvador; Parra, Francisca; L'hermie, Christian y Miquel, M. José (2000). Distribución Comercial, España, Escuela Superior de Gestión Comercial y Marketing, Madrid.

Morgan, Robert M. y Hunt, Shelby D. (1994). The commitment-trust theory of relationship marketing, Journal of Marketing, Vol 58, No 3, United States, American Marketing Association, pp 20-38.

Múgica, José Miguel (1985). Análisis del poder en los canales de comercialización: una revisión de las líneas de investigación, Estudios sobre Consumo, No 4, España, Instituto Nacional de Consumo, pp 82-97.

Narver, John C. y Slater, Stanley F. (1990). The effect of market orientation on business profitability, Journal of Marketing, Vol 54, No 4, United States, American Marketing Association, pp 20-35.

Narver, John C., Slater, Stanley F. y MacLachlan, Douglas L. (2004). Responsive and proactive market orientation and new-product success, The Journal of Product Innovation Management, Vol. 21, No. 5, United States, WileyBlackwell, pp 334-347.

Nonell, Rosa y Borrell, Joan-Ramon (1998). Mercado de medicamentos en España. Diseño institucional de la regulación y de la provisión pública, Papeles de Economía Española, No. 76, España, 
Fundación de las Cajas de Ahorro, pp 113-131.

Ojeda, Yolanda (2009). Informe especial. Se desacelera el pulso, Revista Producto, No. 302, Venezuela, Grupo Editorial Producto, pp 57-64.

Organización Mundial de la Salud - OMS (2009a). Resumen del Análisis de Situación y Tendencias de Salud en Venezuela, en línea: http://www.paho. org/Spanish/DD/AIS/cp_862.htm, fecha de consulta: 04.02.2010.

Organización Mundial de la Salud - OMS (2009b). Situación de la Salud en las Américas: Indicadores Básicos 2009, en línea: http://new.paho.org/hq/index.php?option $=$ com_content\&task= view\&id=1878\& Itemid $=1723, \quad$ fecha de consulta: 04.02.2010.

Podsakoff, Nathan P.; Shen, Wei y Podsakoff, Philip M. (2006). The role of formative measurement models in strategic management research: review, critique, and implications for future research, en D.J. Ketchen y D.D. Bergh (eds.), Research Methodology in Strategy and Management, Vol. 3, Greenwich, JAI Press, pp 197-252.

Poggi, Zulay (2002). Desafíos Tecnológicos de la Industria Farmacéutica Instalada en Venezuela, CENDES, Caracas.

Ramos, Ángel M. y Sanfiel, M. Ángeles (2006). Las relaciones de poder entre la distribución y la industria agroalimentaria en Canarias, XX Reunión Anual ASEPELT, en línea: http://www.fceye.ull.es/asepelt/res_listar_uno.php? num=212, fecha de consulta, 22.05 . 2010.

Reinares, Pedro y Gutiérrez, Emma (2006). Market orientation: Is it accepted as a 21st century marketing strategy in the pharmaceutical industry? An example: Spain, Journal of Medical Marketing, Vol 6, No 4, United Kingdom, Palgrave Macmillan, pp 260-267.
Reinartz, Werner; Haenlein, Michael y Henseler, Jörg (2009). An empirical comparison of the efficacy of covariance-based and variance-based SEM, International Journal of Research in Marketing, Vol. 26, No. 4, Netherlands, Elsevier, pp 332-344.

Ringle, Christian Marc; Wende, Sven y Will, Alexander (2005). SmartPLS 2.0 (M3) Beta, http://www.smartpls.de, University of Hamburg, Hamburg.

Sánchez, Manuel J.; Martín, Félix A. y Rondán F. Javier (2010). Estética y calidad de relación. Una aplicación en los servicios de música online entre jóvenes europeos, Revista Española de Investigación de Marketing ESIC, Vol. 14, No. 1, España, Escuela Superior de Gestión Comercial y Marketing, pp 25-56.

Santesmases, Miguel (1996). Marketing: Conceptos y Estrategias, Ediciones Pirámide, Madrid.

Sanzo, M. José y Vázquez, Rodolfo (2000). Fuentes de conflicto en los canales de distribución industriales: Análisis de los factores condicionantes desde el punto de vista de los distribuidores industriales del sector químico, Dirección y Organización, No. 24, España, Universidad Politécnica de Madrid: Centro de Estudios de Postgrado de Administración de Empresas, CEPADE, pp 107-127.

Solà i Solà, Joaquim (2000). La Indústria Farmacèutica Espanyola: Estructura, Estratègies i Competitivitat, Centre d'economia Industrial, Bellaterra.

Spilker, Bert (1994). Multinational Pharmaceutical Companies, Principles y Practices, Raven Press, New York.

Stern, Louis W.; El-Ansary, Adel I.; Coughlan, Anne T. y Cruz, Ignacio (1999). Canales de Comercialización, Prentice Hall Iberia, Madrid.

Stoltzfus, Timothy (2010). Oversight of marketing relationships between physicians and the drug and device industry: a 
comparative study, American Journal of Law \& Medicine, Vol. 23, No. $2 / 3$, United States, American Society of Law, Medicine and Ethics, pp 326342.

Stone, M. (1974). Cross-validatory choice and assessment of statistical predictions, Journal of the Royal Statistical Society. Series B (Methodological), Vol. 36, No. 2, United States, WileyBlackwell, pp 111-147.

Tenenhaus, Michel; Esposito Vinzi, Vincenzo; Chatelin, Yves-Marie y Lauro, Carlo (2005). PLS path modeling, Computational Statistics \& Data Analysis, Vol. 48, No. 1, Netherlands, Elsevier, pp 159-205.
Tobar, Federico (2008). Economía de los medicamentos genéricos en América Latina, Revista Panamericana de Salud Pública, Vol. 23, No. 1, United States, Oficina Panamericana de Salud, pp 59-67.

Wertheimer, Albert I. (2008). The conflict between the pharmaceutical industry and pharmacists, Journal of Pharmaceutical Marketing \& Management, Vol. 18, No. 2, United States, Taylor and Francis Group, pp 11-19.

Werts, C.E., Linn, R.L. y Jöreskog, K.G. (1974). Intraclass reliability estimates: testing structural assumptions, Educational and Psychological Measurement, Vol. 34, No. 1, United States, Sage, pp 25-33. 